\title{
Le sociologue dans l'action collective face au risque
}

\section{Marc Mormont}

\section{OpenEdition \\ Journals}

Édition électronique

URL : https://journals.openedition.org/developpementdurable/8235

DOI : 10.4000/developpementdurable.8235

ISSN : 1772-9971

Éditeur

Association DD\&T

\section{Référence électronique}

Marc Mormont, "Le sociologue dans l'action collective face au risque », Développement durable et territoires [En ligne], Varia (2004-2010), mis en ligne le 22 juillet 2014, consulté le 28 juin 2022. URL http://journals.openedition.org/developpementdurable/8235; DOI : https://doi.org/10.4000/ developpementdurable.8235

Ce document a été généré automatiquement le 29 septembre 2020.

\section{(c) (7) \&}

Développement Durable et Territoires est mis à disposition selon les termes de la licence Creative Commons Attribution - Pas d'Utilisation Commerciale 4.0 International. 


\title{
Le sociologue dans l'action collective face au risque
}

\author{
Marc Mormont
}

1 Le risque est devenu une préoccupation pour la sociologie. La question posée ici est celle du rôle de l'analyse sociologie dans la prise en compte et la gestion des risques. Nous commencerons par rappeler que le risque est une composante des sociétés industrielles qui ont développé des dispositifs nombreux de gestion du risque. Mais les risques environnementaux, dits «modernes", échappent à ces dispositifs rationalisés d'évaluation et de gestion. Si certains acteurs économiques et politiques continuent de dénier ces risques, on attire ici l'attention sur de nouvelles dynamiques qui s'appuient sur les peurs pour proposer des dispositifs de contrôle. Quelques exemples sont décrits pour montrer que ces dispositifs de contrôle peuvent avoir des effets destructeurs sur des pratiques sociales signifiantes. On fait alors l'hypothèse que ces risques, pas plus que les risques classiques, ne peuvent être réduits par des méthodes d'objectivation qui dénient aux publics un rôle actif, car la gestion des risques est en fait toujours dépendante des conventions qui lient des collectifs vivants. La question est traitée à travers la notion de coordination et des multiples formes qu'elle peut prendre selon la configuration des rapports sociaux. En conclusion, on pose l'hypothèse que la sociologie, plutôt que de répondre à une demande d'objectivation (des perceptions) des risques aurait utilement sa place dans la participation active à une reconfiguration des collectifs et de leurs relations. La recherche-intervention est proposée comme une voie possible.

\section{La société du risque}

Quand U. Beck publie La société du risque en 1986, il introduit dans le débat social et dans la sociologie la question du risque «moderne»; il ouvre à la fois une perspective de recherche sociologique et une interprétation des transformations de la modernité. Deux arguments du propos d'U. Beck introduisent l'analyse qui suit. F. Ewald (1976) avait montré dans l'État Providence combien les sociétés industrielles étaient en fait des sociétés assurantielles, c'est-à-dire qu'un des fondements et une des dynamiques les plus 
importantes de leur développement avait consisté à créer des systèmes d'assurance par rapport aux risques, essentiellement ceux du travail au départ, induits par le développement industriel lui-même. Au fond, les sociétés industrielles sont depuis longtemps des sociétés du risque c'est-à-dire des sociétés où les collectifs productifs s'organisent autour de la gestion du risque. Dans cette interprétation de la société industrielle, le risque est au coeur de la réalité sociale mais la société industrielle a mis au point, développé et progressivement élargi le domaine des risques qui sont induits et pris en charge par des systèmes d'assurance. Et ce système assurantiel a été vertueux puisqu'il a créé non seulement de la sécurité mais aussi une dynamique de progrès technique et d'élargissement des sphères d'activité économique.

3 La prise en charge du risque, dans le modèle de la société industrielle classique, s'inscrit en effet dans la dynamique même du développement, et ce dans deux sens au moins. D'un coté, la création des systèmes d'assurance collective et obligatoire, assortie de la notion clé de responsabilité sans faute (ou responsabilité objective), crée un système d'« éthique sans morale " pour reprendre l'expression de F. Ewald. Les employeurs sont en effet incités à faire de la prévention dans leur propre intérêt et les obligations imposées (de s'assurer collectivement) évitent autant que faire se peut les comportements de free-riding . Nous avons là un beau modèle "historique» d'action collective. D'un autre côté, ce système assurantiel n'est pas seulement fondé sur un paradigme de l'intérêt bien compris : le principe de la responsabilité objective s'appuie aussi sur un principe d'équité, à savoir la protection due au plus faible dans un rapport social salarial forcément très inégal.

4 Une conclusion importante, du point de vue de l'interprétation historique, nous semble être que, contrairement à une vision répandue, le risque est constitutif des rapports sociaux de la société industrielle : il est au cœur des collectifs de travail et des rapports sociaux. Le risque n'est pas extérieur aux rapports sociaux, il en est un constituant dynamique. C'est l'extension progressive des systèmes d'assurance à tous les domaines de la vie qui autorise à parler de sociétés assurantielles.

5 Ce qui change, avec l'émergence de ce que U. Beck appelle "société du risque », c'est qu'un certain nombre de menaces nouvelles apparaissent, menaces qui ne peuvent plus être prises en charge par ces systèmes assurantiels vertueux de l'État Providence. Les risques environnementaux sont évidemment exemplaires de ce qu'il désigne comme «risques modernes». S'ils échappent à la mécanique assurantielle c'est pour deux raisons: d'une part ils concernent des échelles d'espace et de temps qui rendent impossible aussi bien le calcul des dommages (le coût de Tchernobyl est-il évaluable ?) que le calcul des probabilités. Or un système d'assurance moderne est précisément basé sur ces deux données qui permettent de contenir le risque : des instruments de mesure statistique pour prévoir, des modes d'évaluation des dommages pour évaluer la compensation. Les risques que U. Beck dira «modernes » échappent à ces mesures; ils sortent de l'assurable. D'ailleurs les populations concernées par ces risques ne sont pas ou plus identifiables et représentables dans le système assurantiel (générations futures, populations du Tiers-Monde, etc.) et ne peuvent donc ni agir ni être compensées. Car les risques classiques, évaluables, font toujours l'objet d'une négociation sociale complexe. Le risque d'accident du travail conduit en effet à négocier entre employeurs et salariés à la fois le salaire (la prime de risque), la valeur du dommage (l'indemnisation de la victime) et les mesures de prévention (qui touchent l'individu au travail, son savoir-faire, son identité). Or les menaces nouvelles n'ont pas (encore) d'espace de négociation. Cette 
carence est cruciale car elle empêche en fait que se constituent les collectifs de négociation et de gestion du risque. Ces menaces supposent donc la construction d'espaces de négociation nouveaux, inédits. En résumé, et c'est l'argument central de U. Beck, la société industrielle crée des risques nouveaux, de grande ampleur, aux conséquences non calculables, et pour lesquels elles ne peut créer de systèmes assurantiels qui maintiendraient à la fois la cohésion ${ }^{1}$ des collectifs et les dynamiques de création des services et d'innovation qui en découlent et qui sont un des moteurs du développement. En fait ce ne sont plus des risques au sens technique (calculable) mais des menaces qui échappent à la logique du risque assurable et négociable.

6 Voilà le premier argument de U. Beck: les sociétés industrielles sont débordées par des menaces nouvelles, externalités qui ne sont plus traitables et intégrables dans leur propre dynamique. Et ceci conduit au second argument, ces menaces résultent non des aléas naturels, mais de la dynamique même de la société industrielle, de la diffusion des objets techniques, des émissions de polluants, etc., bref de l'activité même des sociétés industrielles.

7 Le second argument de U. Beck, et qui va relier ses analyses à la sociologie des sciences et des techniques, porte sur le dilemme auquel ces nouveaux risques confrontent nos sociétés. Ces nouveaux risques sont les effets, inattendus, imprévus et souvent surprenants, de nos systèmes technico-scientifiques tels qu'ils sont intégrés comme composantes de notre développement. Si ces effets sont inattendus et imprévus, c'est aussi parce que les logiques de la rationalité instrumentale à l'œuvre dans nos systèmes scientifiques et techniques sont des logiques souvent réductionnistes qui s'intéressent à l'efficacité sectorielle et non aux impacts à long terme ou secondaires des techniques. Il considère donc, et rejoint en cela B. Latour d'une certaine manière, que c'est la logique même de la rationalité scientifique et technique qui est au principe de l'émergence des nouveaux risques parce qu'elle rend aveugle aux effets autres que ceux poursuivis immédiatement par les techniques. Mais le dilemme est que ces nouveaux risques ne sont connaissables et appréhendables qu'à travers les outillages techniques et scientifiques. Nous serions donc piégés par des risques que nous ne pouvons découvrir et appréhender qu'à travers les outils mêmes qui ont contribué à les créer. Nous ne pouvons par exemple saisir les effets réels et potentiels et nous prémunir des conséquences de Tchernobyl que grâce aux outils scientifiques et techniques des sciences nucléaires. De plus, les connaissances des risques potentiels sont elles-mêmes insérées dans des systèmes institutionnels qui les cadrent et les orientent si bien que la méfiance devenue légitime à l'égard des institutions se reporte aussi légitimement sur la science et la technique. Cet argument est très important car il met au centre les formes et dispositifs de connaissance et de gestion des risques, et donc la dimension cognitive.

8 Dans la suite de ses travaux, U. Beck (1992) mettra en évidence la nécessité de revoir les arrangements qui articulent science et action publique pour développer leur capacité réflexive, c'est-à-dire leur capacité à penser les effets des constructions cognitives et à intégrer dans la production de connaissances la prise en compte de ces effets. Cela impliquerait une science plus réflexive et plus ouverte sur les attendus sociaux et culturels des recherches, thématique développée par B. Wynne $(1991,1995,2001)$ par exemple en Angleterre.

9 Bon nombre de risques environnementaux, au contraire des risques industriels classiques, sont donc des risques qui ne trouvent pas leurs interlocuteurs pour entrer dans la négociation et la gestion puisqu'il y a des doutes sur les outils cognitifs 
permettant de dire leur réalité, leur ampleur et in fine qui est concerné et en quoi. L'incertitude porte non seulement sur les caractéristiques « objectives » des risques mais aussi sur les institutions chargées de les objectiver, institutions auxquelles participent les scientifiques. Cette double incertitude n'est pas réductible et donc il est difficile de construire les actions collectives de prise en charge de ces risques, car l'action collective et la négociation supposent de pouvoir s'appuyer sur une définition stable du risque et de faire confiance aux modes d'objectivation du risque.

\section{Les risques d'une société de contrôle}

Il ne s'agit pas ici d'attribuer à U. Beck ni a sa notion de société du risque l'interrogation qui s'exprime dans l'analyse suivante mais de dégager les logiques sociales qui se développent à partir de l'idée que notre société est devenue une société du risque. L'idée que nous sommes menacés par des risques potentiellement catastrophiques n'est en effet pas seulement un concept sociologique, une idée de philosophe ; cette idée s'est diffusée, elle est présente dans le monde social et notamment dans les discours de certains activistes environnementaux ; elle prend peu à peu consistance, à juste titre, à partir du diagnostic de risques globaux comme le changement climatique, la prolifération nucléaire, les pandémies d'origine anthropique, les nouvelles technologies, etc. Elle se renforce du constat de nombreuses défaillances des institutions chargées des risques (sang contaminé, etc.). La crainte ou la méfiance à l'égard des productions scientifiques et techniques, si elle n'est pas généralisée, est présente dans nos sociétés à travers des questions comme celle des OGM.

11 La diffusion de l'idée d'une société dangereuse n'est pas sans conséquence. La question qui se pose à partir de l'émergence publique de ces risques environnementaux est alors celle de la manière dont des dynamiques sociales et politiques, puis scientifiques et techniques, se greffent sur ces « représentations » diffuses. Notre hypothèse est que, si on peut observer des débuts de réflexivité à travers des initiatives diverses de dialogue entre science et acteurs sociaux (conférences de consensus, processus dialogiques divers pour reprendre le terme de $\mathrm{M}$. Callon), on observe aussi une autre dynamique que j'appelle dynamique de capture de la peur, dynamique de construction de dispositifs de contrôle (Deleuze, 1990) qui prétendent proposer une réponse technico-scientifique aux peurs que suscitent ces nouveaux risques.

12 Cette dynamique est bien visible, à mon sens, dans les questions de sécurité alimentaire. Les accidents qui se sont répétés ces dernières années dans ce secteur (vache folle, etc.) enclenchent en effet une dynamique de capture de la menace qui procède au recadrage de celle-ci dans des modes d'action qui sont celui du contrôle. Cette dynamique fait intervenir une combinaison de processus médiatiques, marchands, politiques et scientifiques qui s'alignent les uns sur les autres dans un processus qu'on peut styliser comme suit. 0 : un accident ou une alerte grave survient. 1 : les médias s'emparent de l'histoire ou de l'affaire et diffusent une vision potentiellement catastrophique dont peu importe qu'elle soit confirmée dans les faits par la suite car les affaires se succèdent et réalimentent la machine. 2 : les acteurs économiques les plus puissants, notamment industrie et grande distribution, se sentent menacés à court terme (les marchés s'écroulent) et ils prennent des initiatives. À court terme ils cherchent à rassurer et poussent l'État à des mesures radicales de réassurance (par exemple l'abattage de millions de tête de bétail), ce qui produit de nouveaux événements médiatiques. 3 : les mêmes 
acteurs s'adressent à des scientifiques et des laboratoires pour élaborer de nouvelles techniques et surtout pour élaborer des dispositifs de prévention qui sont des dispositifs d'information. 4 : plus rapidement que l'État, car celui-ci est déchiré entre les intérêts en jeu, ils mettent en place des dispositifs nouveaux de contrôle qui vont permettre d'éviter ces crises par exemple via la traçabilité généralisée et via le contrôle strict des pratiques agricoles qui sont désormais les responsables en bout de chaîne. $5:$ les producteurs, ici les agriculteurs, n'ont d'autre choix que de se soumettre à ces dispositifs qui sont à la fois socialement légitimes et fortement équipés en techniques et en formes de coordination (certification, traçabilité, systèmes d'information, procédures, etc.).

13 C'est cette dynamique je veux interroger car elle partage aussi beaucoup de caractéristiques de ce que j'appelle une société de contrôle à savoir une société dans laquelle le contrôle des flux, des circulations, est devenu la clé, et cette société de contrôle ne vise plus la discipline professionnelle ou personnelle mais les seuls flux. Cette société de contrôle (Deleuze, 1990) procède au contrôle via l'information et elle conduit à normaliser les pratiques à partir de ces exigences informationnelles. Cette société de contrôle tend à organiser les pratiques à partir des exigences de sécurité qui sont celles des organisateurs.

14 Je vais en prendre deux exemples. Le premier est anecdotique. La coopérative d'Isigny produit des centaines de milliers de camemberts par an dont une proportion significative de fromages au lait cru. Cette production spécifique est très appréciée des gastronomes, et on se souvient qu'elle a survécu à une réglementation européenne qui voulait pour des raisons d'hygiène interdire toute production à partir de lait cru. On se souvient aussi de la prise de position du prince Charles en faveur de ce camembert au lait cru. C'est que c'est un produit original, basé sur une méthode de production originale, et que beaucoup considèrent comme un patrimoine gastronomique et agricole. Ce fromage est protégé par une Appellation d'Origine Contrôlée (AOC). Au début 2007 un accident survient ; plusieurs enfants ont été atteints d'une infection provoquée par une bactérie inconnue (nouvelle?) présente dans ces camemberts. Ces accidents sont limités, il s'agit notamment de bébés de très jeune âge ( 8 mois). L'affaire est très médiatisée et très rapidement la coopérative d'Isigny suspend la fabrication. L'inquiétant dans cette dynamique, c'est que la décision est fondée sur la crainte du cycle évoqué plus haut. La peur enclenche un mécanisme qui conduit à la destruction à terme d'un système de production. On ne s'interroge pas sur les responsabilités des consommateurs. On n'interroge pas les producteurs sur ce qu'il est possible de faire. La dynamique de la peur est en route.

15 Mon second exemple est moins limité, moins anecdotique. La question des pesticides est typiquement un des ces nouveaux risques. Et l'Union Européenne (UE) a sommé les États Membres de mettre en place un programme d'action en la matière. Différentes stratégies sont possibles. Par exemple, le Danemark a décidé de se donner des objectifs stricts de réduction des quantités de pesticides dans l'agriculture. La Belgique a pris une autre option, très significative, de réduction $d u$ risque lié à l'usage des pesticides. Objectif plus pertinent en apparence puisqu'il est plus précis et semble mieux viser les conséquences, les résultats à atteindre. Mais cet objectif, à première vue généreux, suppose de disposer d'une évaluation des risques. Or les pesticides sont nombreux, utilisés de manière différente selon les cultures, selon le climat, selon les stratégies individuelles etc.. Ils sont donc très différemment présents dans l'espace et dans le temps. Quant aux effets de ces pesticides, on ne dispose que d'indications très partielles de leurs effets dans l'environnement ou sur la santé quand ils se diffusent de manière chronique et à faibles 
doses. Leurs effets à long terme, le long des chaines trophiques, sont très difficiles à analyser. Mais ces difficultés n'arrêtent pas la machine technico-scientifique: il est proposé d'élaborer un «modèle » d'évaluation du risque. Par modèle on entend ici une base de données qui va permettre d'évaluer le risque en tout point du territoire. Comment faire cela? En enchaînant dans le modèle une série d'informations : d'une part les quantités de pesticides de chaque type vendus sur le marché (données faiblement fiables $d u$ fait des usages privés, des échanges transfrontaliers, des réticences de producteurs à diffuser ces informations); d'autre part des données de toxicité qui proviennent des dossiers d'homologation de ces pesticides lors de leur mise en marché, mais ces données sont des données de laboratoire et portent sur des applications ponctuelles à doses élevées : on est obligé d'extrapoler de cela à l'environnement; enfin des données statistiques sur la répartition des cultures dans les communes en faisant l'hypothèse de pratiques uniformes et standards d'application des pesticides dans la culture du blé, dans l'arboriculture, etc. selon les prescriptions des fabricants ou du conseil agricole. On enchaîne ainsi une série de données faiblement fiables mais que le modèle va assembler de manière probabiliste pour définir un niveau de risque. On espère à travers cela définir des mesures qui peuvent aller à l'interdiction de tel pesticide dans telle production... Ce modèle est non seulement faiblement fiable en termes scientifiques mais son usage pour évaluer le risque suppose des choix qui ne relèvent pas de la science ; comme par exemple privilégier soit les fortes doses mais qui sont rares ou au contraire les expositions très fréquentes même si les doses sont faibles. En fait la construction d'un modèle technico-scientifique inclut nécessairement des connaissances mais aussi des choix éthiques. Mais la pratique même de modélisation fait que le débat est enfermé dans le modèle qui devient une boite noire, scientifiquement et socialement. Les producteurs, autant que les consommateurs, en sont exclus.

Ce modèle repose aussi sur un attendu implicite, une croyance extra-scientifique, à savoir que l'industrie sera progressivement capable de remplacer les pesticides actuels par des pesticides moins toxiques ce qui préserve les intérêts des producteurs de pesticides. Cela heurte les agriculteurs car cela impliquera une croissance du coût pour eux..., les nouveaux pesticides étant nécessairement plus chers puisqu'il faut rémunérer l'effort d'innovation.

Moins spectaculaire que le cas du camembert au lait cru, le cas illustre une dynamique de gestion du risque bien caractéristique. Elle procède à la prise en compte prioritaire des enjeux économiques, elle procède par la construction d'une boite noire scientificotechnique, et elle débouche sur une normalisation des pratiques. Elle a aussi pour caractéristique d'ignorer complètement les pratiques des producteurs agricoles et c'est cela qui me paraît faire problème, en ce sens qu'elle procède par ignorance des pratiques et par imposition aux producteurs (et aux consommateurs d'ailleurs) des choix qui sont opérés au risque de détruire ces pratiques et de les rendre aveugles à elles-mêmes.

Ces dispositifs, ces processus de prise en compte du risque, illustrent à mon sens la dynamique d'une société qui met le risque comme catégorie organisatrice de l'action publique. Cette dynamique me paraît potentiellement à la fois aveugle à ses propres effets (en l'occurrence la dynamique des pesticides dans les chaînes trophiques reste inconnue) et destructrice des pratiques agricoles et alimentaires les plus riches par leur diversité et leurs savoir faire. Or la destruction de la diversité des pratiques agricoles, des pratiques de transformation et des pratiques culinaires associées est une menace bien plus grave puisqu'elle conduit à d'autres catastrophes sanitaires. 
19 Par rapport à la notion de société du risque de U. Beck, la question est de savoir quelles conséquences découlent de l'émergence de ces nouveaux risques. La dynamique décrite ci-dessus est une manière pour certains d'en tirer parti. Elle consiste à s'appuyer sur la peur pour constituer le risque comme justification d'une organisation asymétrique des relations. La peur crée, dans les cas évoqués, un collectif imaginaire des victimes potentielles mais elle ouvre à la capture par des acteurs puissants et à la création de collectifs de contrôle et de normalisation des pratiques. Pour élaborer une alternative, on peut développer une approche de l'action collective qui ouvrira à la question du savoir sociologique (des sciences sociales en général) à développer.

\section{Les collectifs de prise en charge du risque}

20 Un système de traçabilité des produits alimentaires est bien une manière de faire un collectif, qui a notamment pour conséquence de déplacer la responsabilité vers le producteur agricole.

\subsection{Action collective : concept}

21 L'action collective peut être définie de plusieurs manières. Dans une perspective sociologique axée sur les mouvements sociaux, elle désigne surtout des formes de mobilisation sociale autour d'un objectif, d'une revendication. Dans une perspective de l'action organisée, elle vise plutôt la production de règles, par un jeu de négociation et de délégation, qui vont conduire à une hiérarchie organisationnelle. Cette perspective est proche de l'analyse économique de l'action collective qui pose également le postulat d'individus dotés d'intérêts, de représentations, de ressources. Mais l'analyse économique met plus l'accent sur la coordination des actions individuelles que sur les jeux stratégiques. Sans entrer dans une longue discussion théorique nous mettrons en avant le concept de coordination dans la mesure où il est relativement neutre quant aux différentes manières dont la coordination peut se faire, par le calcul de l'efficacité (économie), par des valeurs ou objectifs partagés (sociologie), par des traditions (ethnologie) ou par les interactions spatialisées (géographie). Chaque discipline privilégie en fait un mode de coordination comme constitutif de l'action collective et donc un certain nombre de catégories (intérêt, valeur) explicatives. Il est vrai que les rapports marchands sont un mode de coordination qui forme une sorte spécifique d'action collective, mais que la loi ou la tradition sont d'autres formes de coordination qui fonctionnent autrement. La question n'est donc pas de privilège théorique d'une discipline mais plutôt de conformité du modèle d'analyse aux rapports réels de coordination présent dans un champ d'action ou de relations.

\subsection{Action collective et collectifs définis par une pratique}

L'action collective est ici conceptualisée comme le processus de formation des collectifs de formes et de natures diverses, mais où ces collectifs se construisent autour de pratiques finalisées et sensées (ayant du sens pour ceux qu'elles associent). L'action collective est alors définie autrement en sortant du dilemme individuel/collectif d'une part et en se centrant sur les objets de la pratique d'autre part. L'idée de collectifs organisés autour de pratiques traitant d'objets spécifiés par ces pratiques déplace quelque 
peu l'attention de la seule question des rapports sociaux et des règles (typiques des approches sociologique et économiques de l'action collective) vers celle des rapports entre action collective et traitement des réalités sur lesquelles porte l'action collective. Pour le dire sommairement, on n'organise pas de la même manière l'action collective pour régler la circulation sur les routes ou pour de produire une œuvre d'art dans une salle de concert. L'objet de l'action collective importe.

Dans cette perspective, le risque prend une autre signification que dans la lecture précédente ou plus exactement il pousse à questionner le rapport entre pratiques et risque. Le risque, dans la dynamique évoquée ci-dessus, semble une réalité qui s'impose de l'extérieur aux collectifs humains, ce qui tend à établir un rapport d'extériorité de l'événement menaçant. Les sociétés préindustrielles traitaient la menace sur le mode de la punition ou du destin qui, par la médiation des dieux ou des mythes, définissait le collectif humain, orientait son action. C'était une construction du risque, c'est-à-dire une production de la représentation du risque et en même temps de la manière de le traiter pour faire vivre la collectivité qui l'affrontait.

Le risque, quel que soit le contexte, fait nécessairement l'objet d'une construction. En effet, une épistémologie simple permet de poser que le risque n'est pas réel, il n'est pas de l'ordre de ce qui est, mais de ce qui pourrait advenir, donc d'une représentation d'un évènement futur, possible mais qui reste virtuel. Et cette représentation est nécessairement une construction qui passe par des procédures, des outils, des instruments, des jugements quant à ce qui importe et ce qui compte.

Dans les sociétés traditionnelles, cette procédure de construction est souvent de l'ordre du religieux, du mythique, de manière à imputer à des forces extérieures les causes et les explications. La société industrielle telle que thématisée par F. Ewald constitue une manière d'internaliser cette construction dans la perspective d'une maîtrise, mais cette internalisation suppose de rendre la procédure objectivable et discutable, ou plus exactement négociable entre humains (et non plus avec les dieux). Dès lors le risque se construit comme représentation à travers un processus de négociation entre les parties prenantes aux causes et conséquences $d u$ risque si bien que le risque est indissociablement une composante du collectif qui le prend en charge. De ce point de vue, le risque est construit dans un rapport social et reconstruit ce rapport social dans un même mouvement.

Le processus de construction du risque est donc indissociable, mais pas identique, au processus de construction du collectif. En d'autres termes, et nous le savons bien, nous "négocions" en même temps le risque et nos rapport aux autres concernés. Dans la société industrielle il y avait, dans la ligne de F. Ewald, une délégation de certains aspects de la construction du risque à des procédures scientifiques: les statistiques et le calcul des probabilités, l'évaluation des dommages pouvait, non sans tensions et conflits, être déléguée à des spécialistes, à de la technique et de la science et il ne pouvait en être autrement s'agissant par exemple d'évaluer les dommages potentiels d'une inondation ou, jusqu'à un certain point, d'une maladie professionnelle. Nombre d'études ont montré que le paramétrage ou le cadrage de ces évaluations était socialement construit. Mais il faut alors se demander pourquoi le risque n'est pas complètement objectivable. 


\subsection{L'action collective passe par une coordination}

27 C'est ici qu'on peut faire le lien entre action collective comme constitutive de collectifs orientés par des pratiques avec le risque comme construction par des procédures d'objectivation négociées. Le risque comme fait virtuel a notamment deux fonctions : il projette les acteurs dans l'avenir et interroge donc leur existence ou leur permanence, mais aussi il interroge les rapports entre ceux qui sont concernés en tant qu'ils sont parties prenantes, responsables ou victimes, relativement. Définir le risque de contamination alimentaire renvoie nécessairement aux responsabilités distribuées entre producteurs, distributeurs et consommateurs: dans la chaîne du froid, chacun peut défaillir et nous devons penser cette distribution des responsabilités. Associer physiquement un thermomètre au produit de manière à identifier qui a rompu la chaîne est une solution qui permet de résoudre la question de l'imputation des défaillances etc.

La gestion du risque, ou sa prise en compte dans les pratiques, va donc supposer une relation spécifique et nouvelle entre les acteurs concernés. Elle implique une coordination, une action collective et cette action collective, en tant qu'elle est possible ou pas, en tant qu'elle change les rapports entre acteurs, réagit nécessairement sur la représentation du risque. Je pense ici aux paysans qui vivent sur les versants du volcan Galeras en Colombie (Adant et Mormont, 1996). La volonté des autorités de prévenir le risque conduisait à déplacer certains d'entre eux sur d'autres terres, à organiser un réseau de circulation pour évacuer les autres, et tout cela, proposé par des administrations autoritaires, ne pouvait être perçu que comme des agressions, des détours pour les exproprier ou les rendre plus vulnérables aussi bien aux voleurs qu'aux militaires... Leur déni du risque, soutenu par des croyances et des rites religieux de coexistence avec le volcan, était indissociable de leur crainte d'être dépossédé et exproprié et de voir leurs communautés dispersées. Accepter l'existence du risque c'eût été accepter de se soumettre à un danger plus grand encore, celui de dépendre totalement de pouvoirs beaucoup plus concrets et menaçants que celui que signale les grondements occasionnels de la montagne.

En d'autres termes la construction du risque, c'est nécessairement la construction d'un rapport social entre ceux qui le désignent, le gèrent, le préviennent, et organisent tout cela.

De manière plus neutre, pour ne pas dramatiser la mise en scène, le risque n'existe qu'à travers une procédure, des instruments, y compris ceux de la prévention, mais ces instruments postulent eux-mêmes des rapports entre les acteurs. La balance s'effectue entre les coûts de la prise en compte du risque et ceux des événements à risque. L'action collective intervient alors comme le processus à travers lequel la construction du risque se fait de manière à ce qu'il soit pris en compte par des acteurs qui sont concernés par le risque en tant qu'il affecte leurs pratiques. La coordination est possible mais au prix d'une prise en compte simultanée des coûts d'un côté et de l'autre. Ceci est exposé ici en termes de coûts et de bénéfices, dans le langage économique dominant qui facilite la compréhension; mais il peut s'agir évidemment d'enjeux qui ont bien d'autres dimensions qu'économiques, enjeux symboliques ou identitaires, enjeux d'existence ou de survie parfois...

31 Nombre de recherches (Pécaud, 2005 ou pour des exemples Tornatore, 2000 ; Duchêne, 2008) ont montré, de ce point de vue, que la définition des risques, dans le monde du 
travail industriel, comportait cette dimension de négociation multidimensionnelle, c'està-dire mêlant questions d'identité et d'intérêts. Les dispositifs de sécurité sont bien le résultat d'une négociation (inégale bien sûr) entre ce qui est acceptable économiquement, ce qui est faisable techniquement, ce qui est supportable pour le travailleur et ce qui préserve son savoir-faire et son identité. La gestion du risque repose alors sur une coordination qui prend en compte ces multiples dimensions.

Les risques environnementaux nouveaux (climat, perte de biodiversité, etc.) ne peuvent faire l'objet d'une action collective de prise en charge que si les conditions de la coordination sont acquises, à savoir un instrument cognitif de définition du risque et un instrument normatif ou principe de coordination. Ce qui fait leur originalité ou leur caractère critique, c'est précisément que ces conditions ne sont pas données, elles doivent faire l'objet d'une construction.

\subsection{Norme ou principe de coordination et gestion du risque}

La coordination, ou l'action collective s'appuie sur un principe organisateur ou sur la combinaison de plusieurs principes organisateurs. Pensons à la sécurité routière. La coordination, instrumentée par un code réglementaire et par des outils matériels divers (nous y reviendrons), est une coordination pragmatique (rouler à droite ou à gauche importe peu pourvu que tout le monde respecte la règle) mais c'est une coordination qui est quand même complexe. Elle ne peut s'appuyer entièrement sur l'intérêt bien compris d'usagers logiquement intéressés chacun pour soi à éviter les accidents, donc sur la seule logique de l'agrégation spontanée des intérêts: elle suppose une convention au sens le plus élémentaire. Elle doit aussi s'appuyer sur une police qui ne peut se justifier sur le seul impératif de l'efficacité pour chaque individu, elle a besoin d'une justification différente quand on passe à l'échelle de l'action publique qui va se revendiquer d'un intérêt supérieur, à savoir la sécurité publique comme intérêt collectif ou la bonne tenue du trafic pour des raisons d'efficacité collective. C'est ce principe de coordination qui va autoriser et justifier des mesures diverses de coordination et notamment de coercition des activités individuelles via un acteur public ${ }^{2}$.

Il existe différents principes d'action publique comme cadres organisateurs de l'action collective. Par exemple le principe d'équité a pris récemment une (modeste) place dans la coordination de la circulation en attribuant une responsabilité objective aux conducteurs d'automobiles à l'égard des usagers faibles (piétons, cyclistes). Ce principe de coordination s'ajoute aux précédents dans la mesure où, attribuant aux automobilistes une responsabilité plus grande, il enclenche des changements dans les systèmes d'assurance, et, à terme espère-t-on, dans les pratiques des conducteurs à l'approche des passages cloutés. À l'inverse, en distribuant sur tous les assurés le coût des sinistres subis par des habitants en zones inondables, on n'incite guère ni ces habitants, ni les autorités responsables des autorisations de bâtir, à prendre en compte ce risque.

La plupart des systèmes qui se sont stabilisés comme systèmes de définition et de gestion des risques combinent en fait plusieurs principes de coordination et assignent souvent à des instances différentes le soin de préserver chaque principe dans un équilibre pragmatique. Certains risques peuvent être complètement mis en marché et soumis à la loi de l'offre et de la demande, d'autres peuvent être exclusivement attribués à la puissance publique, mais la plupart font la part des choses en essayant de combiner différents principes dans des dispositifs relativement efficaces. 
36 En résumé, l'action collective de prise en charge du risque suppose toujours référence à un ou des principe(s) de coordination qui définissent normativement la forme du collectif. Le choix des principes résulte souvent d'une histoire longue de la constitution sociale des pratiques concernées, ce qui explique la variabilité observée entre les sociétés.

\subsection{Instruments de coordination}

Les instruments et procédures comptent énormément dans ces constructions. Les exemples sont multiples. Comment par exemple imposer à des constructeurs d'automobiles des voitures plus sûres si on ne sait pas évaluer leur résistance aux chocs et les conséquences de ces chocs sur les occupants? Le développement d'une méthode de test (EuroNcap) et leur médiatisation dans un contexte concurrentiel a induit aussi bien une adaptation des produits qu'un développement de normes publiques. La méthode technique de mesure et de comparaison, associée implicitement à un jeu de marché, a bien joué ici comme instrument à la fois d'appréhension du risque et de gestion du risque (en l'occurrence de réduction du risque).

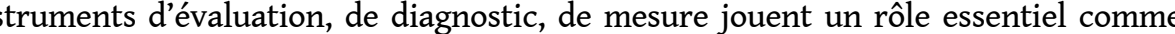
instruments de coordination car ils mettent en forme le risque et permettent de raccorder sa définition aux actions possibles. La plupart des risques ne peuvent être traités sous la seule figure du bien commun, c'est-à-dire d'une menace qui concerne tout un chacun et qui suppose seulement une mobilisation et une coordination simple.

Reprenons à cet égard l'exemple de l'érosion côtière (Herbert et Petit, 2008). Elle menace un certain nombre d'habitations érigées près des plages ou des falaises. Trois stratégies sont possibles; faire des travaux de défense, coûteux pour les pouvoirs publics, de manière à différer l'érosion; mener des actions de prévention en limitant la possibilité de construire dans les zones à risque, reculer c'est-à-dire déplacer les constructions. Pour l'économiste ${ }^{3}$, le choix entre ces stratégies pourrait être fait rationnellement si on pouvait évaluer les coûts de ces stratégies. Mais l'évaluation de ces stratégies suppose de pouvoir prévoir le rythme de l'érosion à moyen terme au moins et aussi de pouvoir actualiser les coûts des constructions perdues au fil du temps. Si cela était possible, on pourrait à travers un analyse coût-bénéfice par exemple choisir, dans certains cas, d'indemniser quelques propriétaires (au nom d'un principe d'équité) en les déplaçant plutôt que d'entreprendre des travaux de protection très coûteux ce qui ne correspondrait plus à un principe d'efficacité. On pourrait aussi mieux définir les coûts de la prévention mais celle-ci devrait être mise en regard avec les bénéfices du développement touristique. L'incertitude est à la fois « naturelle » et sociale. En l'absence d'un instrument capable de définir les coûts des stratégies et d'arbitrer en recourant à un principe de coordination acceptable, le conflit est fort et l'action collective bloquée.

Dans ce type de situation, l'action collective manque non seulement de principes de coordination mais aussi des outils cognitifs de définition du risque. On voit bien que les principes de coordination ne sont pas évidents ; faut-il laisser la responsabilité de leurs choix aux individus, aux constructeurs et laisser jouer la loi du marché et du calcul individuel ? Ou faut-il faire jouer le principe d'équité et indemniser les victimes, au moins celles qui se sont installées avant le processus d'évaluation du risque ? Ou encore faut-il aussi prendre en compte les intérêts collectifs locaux, à savoir les intérêts du secteur touristique, principale source de revenus de la région? Le choix du principe de coordination ou de la combinaison des principes d'arbitrage renvoie en fait à la 
composition des collectifs qui sont intéressés au risque et à sa gestion. Or la composition du collectif ou des collectifs pris en compte est de nature à modifier l'instrument, c'est-àdire le calcul des coûts des stratégies. Mesurer les impacts indirects d'une limitation du développement touristique sur la région élargit, spatialement, temporellement et socialement le collectif concerné... Ces différentes hypothèses laissent entrevoir ce qui est l'argument défendu ici : il n'y a pas de logique nécessaire qui va de la nature d'un risque aux normes de sa gestion, pas de one best way. La manière dont le risque est défini cognitivement dépend de ce qui est pris en compte dans l'opération de définition ; mais inversement la connaissance du risque va contribuer à définir les frontières des collectifs concernés et va se prêter à l'usage de tel ou tel principe de coordination. Si on parle de construction sociale du risque, c'est bien pour indiquer ce processus qui fait interagir outils cognitifs, normes de coordination et collectifs.

Il s'ensuit deux conséquences importantes. La construction du risque se fait à travers des instruments qui dépendent des collectifs concernés mais ces instruments, associés à des principes de coordination, rendent possible ou non la définition d'un collectif capable de prendre le risque en charge, de le "gérer ». Un des processus importants est ici constitué par les anticipations que les acteurs vont faire en se référant à la fois aux connaissances du risque et aux normes instituées.

Ceci positionne alors la démarche scientifique de mesure et d'évaluation : d'une part elle dépend des collectifs qui se présentent comme à prendre en compte, et d'autre part elle propose des instruments permettant de constituer ou de reconfigurer l'action collective. Différents cas de figure se présentent alors selon que le risque émerge d'une action collective de mobilisation de victimes, ou selon que le risque vient de l'extérieur se proposer comme une exigence de prise en compte par des collectifs existants; selon que les collectifs concernés sont organisés par tel ou tel principe de coordination. Toute démarche scientifique d'objectivation du risque constitue alors un élément qui peut intervenir dans la construction du risque et ceci pose la question des modes d'intervention de la science, et notamment pour leur part des sciences humaines.

\section{L'intervention du sociologue}

43 La spécificité des risques environnementaux, dans la théorisation de U. Beck, c'est que ce sont des menaces qui, induites par l'activité humaine, ne sont cependant pas inscrites dans le fonctionnement des collectifs. Elles résultent d'externalités, d'effets oubliés ou négligés par le collectif. Elles le débordent. Ni l'entreprise productrice d'amiante, ni le marché, ni les consommateurs ne voyaient ou ne voulaient voir ce risque. Il émerge de l'extérieur des collectifs organisés, souvent à partir de victimes qui s'organisent ou à partir d'alertes scientifiques. Le cas de figure est alors généralement celui de collectifs à qui on oppose le risque tel qu'il se construit de l'extérieur par des procédures technicoscientifiques. Et on se heurte souvent à des attitudes qui vont du déni du risque à l'amplification du risque. Nous interrogeons alors le rôle de la recherche sociologique dans ces affaires.

Le plus souvent ce qui est demandé au sociologue c'est de travailler à éclairer les perceptions du risque, ou encore les représentations du risque. Cette demande, émanant souvent d'acteurs puissants (État, entreprises, scientifiques organisés), repose habituellement sur le postulat d'une irrationalité populaire face au risque, cette irrationalité étant opposée à la définition objective du risque par la science. Elle reflète 
déjà une structuration du champ social qui fait du public une instance privée des outils de connaissance du risque. Cette demande attribue alors au sociologue un rôle d'objectivation : il doit dire ce en quoi les acteurs se trompent et les raisons objectives de l'erreur qu'on peut espérer corriger. Cette manière de faire contribue à naturaliser les publics, à en faire de simples objets susceptibles d'explication (et espère-t-on agissables). Mais la plupart des études de représentations du risque ont été des échecs et n'ont guère donné lieu à des politiques robustes. C'est précisément parce que, naturalisant les rapports aux risques ${ }^{4}$, elles sont fragiles et souvent sans effet sur l'action collective qui continue et qui transforme le rapport au risque qui n'est jamais seulement un rapport de représentation.

On peut essayer de définir autrement l'intervention du savoir sociologique en admettant précisément le lien fort entre constitution du collectif et risque. L'hypothèse pourrait être formulée comme suit: la connaissance sociologique peut intervenir comme une des connaissances constitutives du collectif (comme connaissance de soi et connaissance d'autrui) c'est-à-dire ayant comme possibilité de contribuer à reconfigurer le collectif, dans certaines conditions et dans certaines limites. Il ne s'agit pas de faire du sociologue le démiurge de la société du risque, mais de s'interroger sur la manière dont la connaissance que les collectifs ont d'eux-mêmes (ou peuvent acquérir) peut agir. Dans cette hypothèse le sociologue doit se considérer comme intervenant et non comme expliquant, doit considérer ses hypothèses et théories non pour ce qu'elles expliquent mais pour ce qu'elles contribuent à produire, sans qu'il puisse pour autant s'attribuer ni vouloir jouer un rôle prédominant dans l'action.

Il est tout à fait possible d'admettre comme point de départ que, dans une situation, quelle qu'elle soit, les acteurs sont déjà organisés, c'est-à-dire qu'ils disposent déjà d'un principe et d'outils de coordination qui sont mis à leur disposition par les équipements multiples à disposition dans nos sociétés. Prenons le cas d'un ensemble de riverains confrontés à une pollution accidentelle ou chronique qui leur est révélée par une alerte ou par les médias : la référence à la santé fortement liée à un principe d'équité et la communication médiatique forment les premières ressources sur lesquelles entamer une action; celle-ci peut alors se déployer en mobilisant les rapports de voisinage, la communauté politique locale, mobilisant ainsi d'autres principes de coordination et utilisant d'autres instruments (manifestations, tracts). Stratégiquement il est logique que ces victimes potentielles d'un risque qui leur est imposé le mettent en exergue, l'amplifient et fassent pièce à toute tentative et tentation de déni de la part de l'industriel ou de l'autorité politique. Se constitue ainsi un collectif d'action, une action collective si on veut, qui va se stabiliser dans une forme d'organisation (un comité local, une association de défense). Tout autre est sans doute la forme d'action collective d'un collectif de travailleurs qui font un travail dangereux dans une entreprise quelconque. Il est vraisemblable qu'un tel collectif, qui affronte autrement un risque, concilie un principe d'efficacité du travail et un principe de solidarité de groupe (indispensable à leur travail). Ceci peut se faire à travers des instruments techniques et des règles de conduite et cela conduit quelquefois à des attitudes de déni ou de sous-estimation du risque ${ }^{5}$, à des pratiques de ritualisation, à des rites d'initiation des nouveaux, etc., autant d'instruments de coordination de l'action collective dans le travail.

Dans ces deux exemples il est clair que l'analyse sociologique qu'on peut faire ne peut rester sans effet sur les groupes concernés. Dans le premier cas, si elle se centre par exemple sur les appuis de l'action collective des riverains, elle peut très bien fragiliser 
celle-ci en montrant la faiblesse des informations scientifiques qui justifient l'action. Dans le second cas, révéler les tactiques par lesquelles les travailleurs s'organisent informellement pour maintenir leur autonomie de travail, quitte à contourner des mesures de sécurité, peut très bien donner prise à un pouvoir pour réaligner leurs pratiques. Les acteurs disposent (heureusement) de tactiques pour éviter ces emprises sur leur action. Mais cela pose la question de l'effet possible des connaissances sociologiques sur les situations «à risque ». De même, la production de connaissances économiques sur la manière dont les gens se situent par rapport aux risques (via des évaluations contingentes ou des méthodes de prix hédoniques) peuvent faire l'objet d'interprétations qui vont être mobilisées dans l'action collective. Ainsi l'analyse des fluctuations des prix des logements selon la distance à la source de la pollution peut-elle devenir un argument pour ceux qui réclament des compensations économiques (s'ils se réfèrent à un principe de coordination par le marché) ou au contraire qui demandent une prise en charge sanitaire (s'ils mobilisent plutôt un argument d'équité sociale). La connaissance scientifique ne produit donc pas nécessairement d'effet par elle-même mais elle peut être une ressource pour l'action collective.

Dès lors l'intervention sociologique, nous donnons ici un sens large à ce terme pour désigner toute forme d'engagement du sociologue dans la situation, requiert-elle un positionnement du chercheur par rapport à cette action collective. Le positionnement de la recherche-action représente une solution. Dans ce cas le sociologue s'attache à une action collective, à un groupe et il s'attache à une transformation du groupe et de ses rapports aux autres acteurs. La recherche-intervention est une autre piste dans laquelle le sociologue se posera plutôt en médiateur, en intermédiaire entre les acteurs dans la recherche d'autres formes de coordination.

Dans la recherche-intervention (Stassart et Mormont, 2008) telle que nous l'entendons la tâche du sociologue est alors moins de rendre compte des formes que prennent les coordinations et les instruments que de rechercher, avec les acteurs, des formes de coordination nouvelles qui puissent faire évoluer la situation. Cette posture de recherche positionne le sociologue comme le co-auteur d'un diagnostic mené avec des acteurs d'une situation. Ce diagnostic consiste à faire émerger les différents points de vue sur la situation, à rendre explicites les divergences voire les tensions par exemple dans la définition du risque ou des responsabilités. L'intervention comporte donc une dimension cognitive de mise à jour de cette situation et d'un diagnostic partagé. Mais elle comporte aussi une dimension pratique qui est de transformer les relations entre les protagonistes. Le travail consiste alors à élaborer un programme d'action et de recherche sur lequel peuvent s'accorder les acteurs: il s'agit de produire des connaissances mais aussi de modifier les relations entre les acteurs, les deux démarches étant indissociables. Ce dont il s'agit c'est donc de produire des capacités collectives (ce qui ne veut pas dire communes) de prise en charge du problème. Dans ce processus il ne s'agit plus pour le sociologue de dire, dans une perspective déterministe, la vérité des acteurs, de leurs motivations ou déterminations, mais bien d'explorer quelles redéfinitions de leurs pratiques, de leurs compétences et de leurs relations sont possibles et à quelles conditions. 


\section{Conclusion}

50 collectifs concernés ne sont plus fixées et ne peuvent l'être, la question de l'action collective se pose comme un défi aux sciences sociales qui ne peuvent plus se contenter de la position d'observateur. processus de prise en charge de ces risques, processus dont la dynamique est aussi inquiétante que les risques qui les suscitent. On peut même se demander si la dynamique de la peur, dont on a fait l'hypothèse qu'elle se développe dans nos sociétés indépendamment de l'évaluation du risque, ne concerne pas même des risques tout à fait classiques (c'est-à-dire évaluables et assurables) comme certains risques alimentaires. Une telle dynamique de la peur, si elle se met à envahir le champ social, est une dynamique vertueuse si elle porte l'attention sur les menaces, mais c'est une dynamique perverse et dangereuse si elle conduit à priver les acteurs de toute prise sur le risque. Or j'ai fait l'hypothèse de ce que j'ai appelé la capture de la peur pour indiquer que certains acteurs peuvent s'appuyer sur elle pour imposer des formes de contrôle qui, ayant ou non une prise réelle sur le risque, conduisent à normaliser (au mieux) ou à détruire des pratiques signifiantes. Il y a là une dynamique de l'action collective, dominée par les rapports marchands, où l'individu (consommateur) est la référence, le prétendu décideur auquel la sécurité est due par tous les moyens. Cet individu privé (de toute compétence) permet de justifier une emprise d'instances de contrôle qui promettent, par la technologie ${ }^{6}$ conjuguée au marché, cette sécurité.

Si on partage au moins un peu cette inquiétude face à cette dynamique, le savoir sociologique ne peut se limiter à démonter et dénoncer cette dynamique, au risque d'arriver toujours trop tard pour voir se confirmer les hypothèses pessimistes qu'on pouvait faire. Le développement durable appelle un autre positionnement, notamment le choix, si un risque grave est avéré, de développer des connaissances permettant de créer l'action collective capable d'empêcher cette virtualité. Cela demande non seulement un positionnement éthique, mais aussi une autre épistémologie du risque. En bref on ne peut se résoudre au constat du détournement de la peur et de l'impuissance face aux menaces collectives, et il faut créer des connaissances et des méthodes qui permettent de constituer des collectifs de gestion du risque capables en même temps de préserver l'existence vivante de ces collectifs.

BIBLIOGRAPHIE

Adant I. et Mormont M. (1998), « Quand prévention rime avec menace : le volcan Galeras en Colombie », Environnement et Société, $n^{\circ} 21: 27-60$

Beck U. $(2001,1986)$ La société du risque. Sur la voie d'une autre modernité, Paris, Éditions Aubier ; édition originale : Risikogesellschaft, Francfort, Suhrkamp Verlag 
Beck U. (1992), From Industrial Society to Risk Society : Questions of Survival, Social Structure and Ecological Enlightenment, Theory, Culture and Society, vol. $9: 97-123$

Deleuze G. (1990), Post-scriptum sur les sociétés de contrôle, in Pourparlers, Paris : Editions de Minuit : 240-247

Duchêne F. (2008), « Les affaissements miniers dans le bassin ferrifère lorrain : quand le territoire re-politise la gestion du risque ", Développement durable et territoires [En ligne], Dossier 11 :

Catastrophes et Territoires, Consulté le 21 janvier 2009. URL : http://

developpementdurable.revues.org/index7073.html

Ewald F. (1976), L'État Providence, Paris : Grasset

Herbert V. et Petit O. (2008), Risque environnemental et action collective. Application aux risques industriels et d'érosion côtière dans le Pas-de-Calais, Lille : IFRESI, juillet, $122 \mathrm{p}$

Pecaud D. (2005) Risques et précaution. L'interminable rationalisation du social, Paris : La Dispute, $314 \mathrm{p}$

Stassart P. et Mormont M. (2008), « La recherche-intervention pour une transition vers le développement durable », Economie Rurale.

Tornatore J.-L. (2000), « Leçon de sociologie des paysans-mineurs du Briançonnais », in Rabier J.C. (2000), Anthropologie du bassin minier. Formes de mobilisation. Actes du colloque international de Béthune , Lille, IFRESI : 309-334

Wynne B. (1991), “Knowledges in context". Science, Technology \& Human Values 16 (1) : 111-121

Wynne B. (1995), “Technology assessment and reflexive social learning: Observations from the risk field”, in Rip, A., Misa, Th. and Schot, J. (Eds.) (1995), Managing Technology in Society. The Approach of Constructive Technology Assessment, Pinter Publishers, London

Wynne B. (2001), "Managing scientific uncertainty in public policy", paper presented at the " Biotechnology and Global Conference: Crisis and Opportunity" (26-28 April), Cambridge

\section{NOTES}

1. Il ne s'agit pas de nier que les risques professionnels ont fait et continuent de faire l'objet de luttes sociales très vives, mais ces luttes sont intégrées à la dynamique de développement des sociétés industrielles.

2. L'action collective ainsi entendue comprend donc plusieurs dispositifs emboités : un premier niveau est celui de la coordination des conduites individuelles dans le trafic, laquelle est possible grâce à des instruments de coordination (conventionnels) sur fond d'une norme implicite d'intérêt personnel, mais le manque de discipline spontanée appelle un deuxième dispositif de délégation de la surveillance à l'État et sa police, ceci s'appuyant sur des normes collectives.

3. Il faut reconnaître à l'économie d'avoir mis en évidence et tenu comme programme de recherche cette question de la coordination. Il faut cependant se tourner vers les économies dites « hétérodoxes» pour trouver une analyse de la coordination via des principes et des instruments autres que marchands.

4. A l'inverse quelques études ont réussi à montrer au moins que ce n'était pas l'incompétence ou l'ignorance qui étaient au principe de rejet de certaines innovations, spécialement dans la controverse sur les OGM.

5. On sait par expérience commune que la maîtrise pratique de situations à risque suppose, dans la pratique, une attention aux gestes qui implique une mise au second plan du risque lui-même. 
6. Il faudrait insister que ces «technologies" ne nécessitent pas des fondements scientifiques solides, il leur suffit de traiter des données, des corrélations pour se donner une possibilité de contrôle. Elles rejoignent en cela les nouvelles technologies du génome.

\section{RÉSUMÉS}

Le risque est une composante centrale des sociétés industrielles modernes qui en ont organisé la prise en charge à travers une série de dispositifs complexes. Les questions environnementales font émerger de nouveaux risques dits modernes parce qu'ils échappent à ces dispositifs d'évaluation et de gestion. A côté d'attitudes de déni de ces risques on voit apparaitre des dynamiques qui sont vues comme des dispositifs de capture des peurs qui ont pour effet de donner une emprise forte à ceux qui peuvent mettre en place des dispositifs de contrôle, qui peuvent détruire les collectifs organisés autour de pratiques vivantes. Le sociologue ne peut plus se contenter de répondre à une demande d'analyse des perceptions des risques; il doit s'engager dans l'action collective qui confronte les collectifs aux risques pour aider à leur reconfiguration tout en préservant leur autonomie.

Risk is a central component of modern industrial societies that have organised complex systems to tackle with them. Environmental problems progressively reveal new risks that are said modern risks because they can be dealt with through usual systems of risk assessment and management. If some authorities still deny these risks, it is important to observe new dynamics that are here described as capture of fear and that are giving strong power to those who are able to propose control systems. These systems can be destructive of many original and creative practices. Sociologist can not be confined in analysis of risk perception, he has to be involved in collective action that try to deal with risks by confronting different collectives and by reorganising them while preserving autonomy.

\section{INDEX}

Keywords : coordination, fear, intervention research, organisation, risk

Mots-clés : coordination, peur, collectif, recherche-intervention, risque

\section{AUTEUR}

\section{MARC MORMONT}

Marc Mormont est chercheur en sociologie au SEED/Université de Liège 